\title{
Clinical Utility of a Novel Ultrafast T2-Weighted Sequence for Spine Imaging
}

\author{
(D) M.B. Keerthivasan, (D) B. Winegar, (D).L. Becker, (D) A. Bilgin, (D) M.l. Altbach, and (D) M. Saranathan
}

\begin{abstract}
BACKGROUND AND PURPOSE: TSE-based T2-weighted imaging of the spine has long scan times. This work proposes a fast imaging protocol using variable refocusing flip angles, optimized for blurring and specific absorption rate.
\end{abstract}

MATERIALS AND METHODS: A variable refocusing flip angle echo-train was optimized for the spine to improve the point spread function and minimize the specific absorption rate, yielding images with improved spatial resolution and SNR compared with the constant flip angle sequence. Data were acquired from 51 patients (35 lumbar, 16 whole-spine) using conventional TSE and the proposed sequence, with a single-shot variant for whole-spine. Noninferiority analysis was performed to evaluate the efficiency of the proposed technique.

RESULTS: The proposed multishot sequence resulted in a $2 \times$ shorter scan time with a $>1.5 \times$ lower specific absorption rate. The variable flip angle sequence was noninferior to the conventional TSE $(P<.025)$ for all image-quality and clinical criteria except signal-to-noise ratio for the lumbar spine protocol. However, mean image scores for the TSE-variable refocusing flip angle were $\geq 4.3$ for all criteria, and concordance analysis showed high agreement (>90\%) with the TSE, indicating clinical equivalence. The single-shot sequence resulted in $4 \times$ shorter whole-spine scans, and image scores were $\geq 4.4$ for all criteria, attesting to its clinical utility.

CONCLUSIONS: We present a fast T2-weighted spine protocol using variable refocusing flip angles, including a single-shot variant. The sequences have better point spread function behavior than their constant flip angle counterparts and, being faster, should be less sensitive to patient motion, often seen in the longer TSE scans.

ABBREVIATIONS: $E T L=$ echo-train length; $S A R=$ specific absorption rate; $V F A=$ variable refocusing flip angle

$\mathbf{T}$ 2-weighted MR imaging protocols are routinely used in the clinic for spine imaging. T2WI sequences such as $2 \mathrm{D}$ fast spinecho or turbo spin-echo have proved useful for the detection and diagnosis of osteomyelitis, stenosis, nerve root compression, disc characterization, and other pathology. ${ }^{1,2}$ A typical spine protocol includes sequences for T2WI, T1WI, and short-tau inversion recovery fat-suppressed T2 imaging, all with FSE/TSE readouts. While TSE/FSE with multiecho readout reduces scan times compared with conventional spin-echo sequences, the scan times are still on the order of 3-4 minutes, and these double or triple when

Received September 22, 2017; accepted after revision May 14, 2018.

From the Departments of Electrical and Computer Engineering (M.B.K., A.B.), Medical Imaging (M.B.K., B.W., J.L.B., M.I.A., M.S.), and Biomedical Engineering (A.B.) University of Arizona, Tucson, Arizona.

Paper previously presented, in part, at: Annual Meeting and Exhibition of the International Society for Magnetic Resonance in Medicine, April 22-28, 2017; Honolulu, Hawaii.

Please address correspondence to Manojkumar Saranathan, PhD, Department of Medical Imaging, University of Arizona, 1609 N Warren Ave, Tucson, AZ 85724

e-mail: manojsar@radiology.arizona.edu

http://dx.doi.org/10.3174/ajnr.A5713 performing combination spine examinations (eg, thoracolumbar). This scan time is uncomfortable for patients with painful spine conditions and makes the imaging more vulnerable to motion artifacts. While approaches using parallel imaging ${ }^{3}$ have been explored to reduce scan times, the use of higher acceleration factors is limited by image-quality degradation arising from poor coil coverage. While the scan time of a multislice TSE sequence could also be reduced by increasing the echo-train length (ETL), the corresponding increase in the specific absorption rate (SAR) results in an increased minimum $\mathrm{TR}$, which offsets any reduction in scan time. In addition, increased ETL exacerbates image blurring from T2 decay during the long echo-train.

Single-shot sequences such as single-shot fast spin-echo and HASTE have been used for lumbar spine myelography, ${ }^{4}$ cervical spine motion imaging, ${ }^{5}$ and functional imaging of the spine. ${ }^{6}$ However, these sequences are not preferred for routine T2WI because they have severe image blurring and SAR limitations arising from the long echo-trains, often addressed by scanning at a low spatial resolution. ${ }^{7}$

Various approaches have been proposed to minimize radio- 
frequency power deposition in TSE-based sequences. ${ }^{8-10}$ These approaches optimize the refocusing flip angle train to improve the imaging point spread function, leading to reduced T2 blurring and an improved signal-to-noise ratio and SAR performance. While the variable refocusing flip angle (VFA) technique has been successfully used for 3D TSE-based brain, ${ }^{11,12} \mathrm{knee},{ }^{13,14}$ and spine $^{15}$ imaging, its use in $2 \mathrm{D}$ imaging has been rather limited. Recently, VFA along with 2D single-shot TSE was used in abdominal and pelvic imaging ${ }^{16}$ with increased sharpness and significant reduction in scan times due to the reduced SAR compared with conventional single-shot TSE.

In this work, we propose a fast $\mathrm{T} 2$-weighted spine imaging protocol using a multishot turbo spin-echo sequence with variable refocusing flip angles (TSE-VFA) optimized for spatial resolution, SNR, and SAR. We also explore the use of a single-shot variant (HASTE-VFA) as a fast T2WI screening sequence for whole-spine protocols, in which the use of conventional TSE is prohibitively long.

\section{MATERIALS AND METHODS}

\section{Pulse Sequence and Parameter Optimization}

The vendor-supplied TSE and HASTE sequences were modified to incorporate the refocusing flip angle modulation scheme proposed by Busse et al, ${ }^{10}$ which parameterizes the refocusing flip angle train by using 4 control angles $\left(\alpha_{\text {start }}, \alpha_{\text {min }}, \alpha_{\text {cent }}, \alpha_{\text {end }}\right)$. Following the approach of Loening et al, ${ }^{16}$ flip angles were optimized to maximize the SNR for the desired effective TE and minimize the SAR, with minimal spatial blurring. Numeric simulations were performed using Matlab (MathWorks, Natick, Massachusetts) to narrow the range of "optimal" refocusing flip angle values. For each possible value of the control angle vector ( $\left.\alpha_{\text {start }}, \alpha_{\text {min }}, \alpha_{\text {cent }}, \alpha_{\text {end }}\right)$, T2 signal evolution from the turbo spinecho pulse sequence was simulated using the Echo Phase Graph algorithm, assuming $\mathrm{T} 1$ and $\mathrm{T} 2$ values for the spine at $3 \mathrm{~T}$ ( $\mathrm{T} 1=$ $1060 \mathrm{~ms}, \mathrm{~T} 2=69 \mathrm{~ms}$ ), echo-train length $=56$, and effective $\mathrm{TE}=$ $105 \mathrm{~ms}$. The imaging point spread function was computed using the simulated signal. The peak and full width at half maximum value of the point spread function were measured because these are indicative of the SNR and resolution, respectively. Relative SAR was computed as the sum of squared refocusing flip angles divided by the echo-train length: $S A R_{\text {rel }}=\frac{\sum_{\mathrm{i}=1}^{E T L} \alpha_{\mathrm{i}}}{E T L}$, where $\alpha_{\mathrm{i}}$ is flip angle of the ith refocusing pulse.

The optimal flip angles were chosen by using a 2-step search algorithm: 1) The search space was reduced by choosing control angles that maximize the point spread function on the basis of a preset threshold while maintaining the SNR at the effective TE. 2) Control angles that yield minimum SAR were chosen to generate the tailored refocusing flip angle train. This approach ensures adequate resolution and SNR for the VFA sequence compared with a constant flip angle train for the same echo-train length. To minimize motion-related signal dephasing at very low values of $\alpha_{\min }{ }^{17}$, we chose a value of $\alpha_{\text {min }}=45^{\circ}$. Due to the nonconvex nature of the search space, the resultant flip angle parameters are one of many possible optimal solutions.
Table 1: Scan parameters used for the conventional TSE and the proposed variable flip angle sequences

\begin{tabular}{|c|c|c|c|}
\hline Parameters & $\begin{array}{c}\text { Conventional } \\
\text { TSE }\end{array}$ & TSE-VFA & HASTE-VFA \\
\hline $\begin{array}{l}\text { Resolution (phase } \times \\
\text { freq) }\left(\mathrm{mm}^{2}\right)\end{array}$ & $0.81 \times 0.73$ & $0.81 \times 0.73$ & $1.25 \times 1.0$ \\
\hline Slice thickness (mm) & 3 & 3 & 3 \\
\hline Refocusing flip angle & $140^{\circ}$ & $\begin{aligned} \alpha_{\text {start }} & =130^{\circ} \\
\alpha_{\text {min }} & =45^{\circ} \\
\alpha_{\text {cent }} & =110^{\circ} \\
\alpha_{\text {end }} & =45^{\circ}\end{aligned}$ & $\begin{aligned} \alpha_{\text {start }} & =130^{\circ} \\
\alpha_{\text {min }} & =50^{\circ} \\
\alpha_{\text {cent }} & =90^{\circ} \\
\alpha_{\text {end }} & =45^{\circ}\end{aligned}$ \\
\hline $\begin{array}{l}\text { Parallel imaging } \\
\text { acceleration } \\
\text { factor }\end{array}$ & 2 & 2 & 1 \\
\hline ETL & 21 & 56 & 160 \\
\hline TR (ms) & 2800 & 3600 & 770 \\
\hline Scan time (min) & $3 \mathrm{~min} 25 \mathrm{sec}$ & $1 \mathrm{~min} 28 \mathrm{sec}$ & $1 \mathrm{~min} 57 \mathrm{sec}$ \\
\hline
\end{tabular}

Note:-freq indicates frequency.

\section{Phantom and Volunteer Scanning}

TSE-VFA and HASTE-VFA sequences were implemented and tested on a 3T Siemens Skyra scanner (Siemens, Erlangen, Germany). Phantom experiments were performed to study the blurring behavior and SNR of the proposed technique based on flip angle parameters chosen from simulations. Two nickel-doped agarose gel phantoms with $\mathrm{T} 2=45 \mathrm{~ms}, \mathrm{~T} 1=1000 \mathrm{~ms}$ and $\mathrm{T} 2=$ $80 \mathrm{~ms}$, T1 $=1300 \mathrm{~ms}$ were imaged using the following sequences: a conventional TSE sequence at ETL $=21, \mathrm{TSE}$ at $\mathrm{ETL}=56$, and TSE-VFA at ETL $=56$. Data were acquired at an effective $\mathrm{TE}=100 \mathrm{~ms}$ for all 3 sequences. The mean signal in the 2 phantoms was measured by drawing ROIs inside the object and in the background noise. The acquisition SNR was computed as $S N R=\frac{\text { Signal }_{\text {mean }}}{\text { Background }_{\mathrm{SD}}}$. In addition, SNR efficiency was calculated as $S N R_{\text {eff }}=\frac{S N R}{\sqrt{\text { Scan Time }}}$. The use of variable refocusing flip angles can alter TSE image contrast. To ensure comparable contrast behavior of TSE-VFA, we also measured relative contrast between the 2 phantoms: RelC $=\frac{\left(\text { Signal }_{1}-\text { Signal }_{2}\right)}{\text { Signal }_{1}}$. The SAR computed by the scanner was also recorded for the 3 sequences.

Because a complete sweep of the flip angle parameter space is not practical in vivo, the optimal flip angles obtained from simulations were validated using data acquired from volunteers. Sagittal lumbar spine data were acquired from 5 volunteers after informed consent, using the conventional TSE and TSE-VFA sequences with the parameters listed in Table 1.

\section{Clinical Imaging}

The optimized TSE-VFA sequence was added to the lumbar and cervical spine protocols as an addition to the routine T2WI TSE sequences. The HASTE-VFA sequence was added to the wholespine protocol (covering the cervical, thoracic, and lumbar spine), which currently omits the T2WI TSE sequence due to long scan times ( $\sim 9$ minutes). This omission was possible due to the lower spatial resolution typically used in whole-spine screening protocols, which makes single-shot VFA with an adequate SNR viable. The effective TE was set to $108 \mathrm{~ms}$ for all the sequences, and images were acquired in the sagittal plane in accordance with the standard clinical protocol. The scan parameters for conventional 

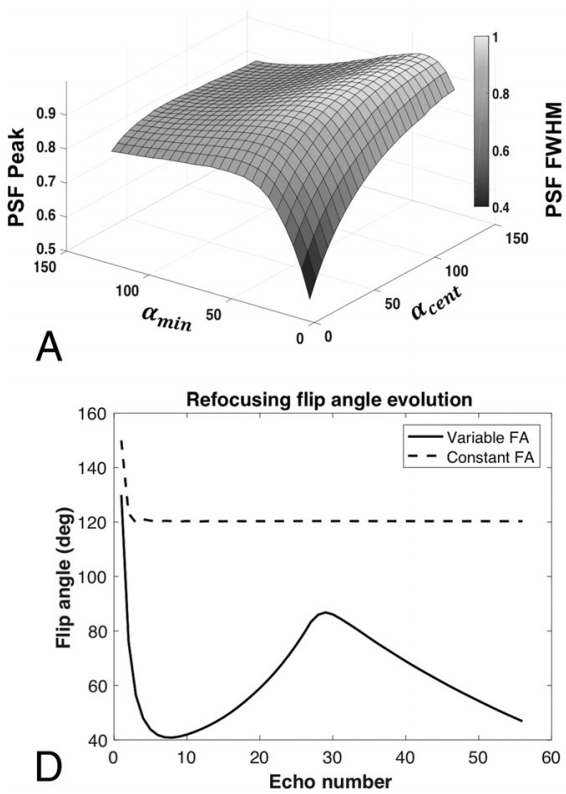
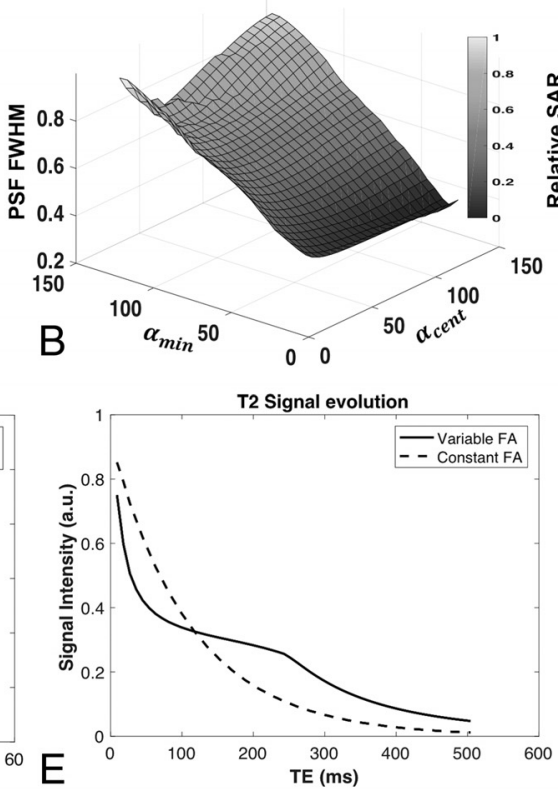
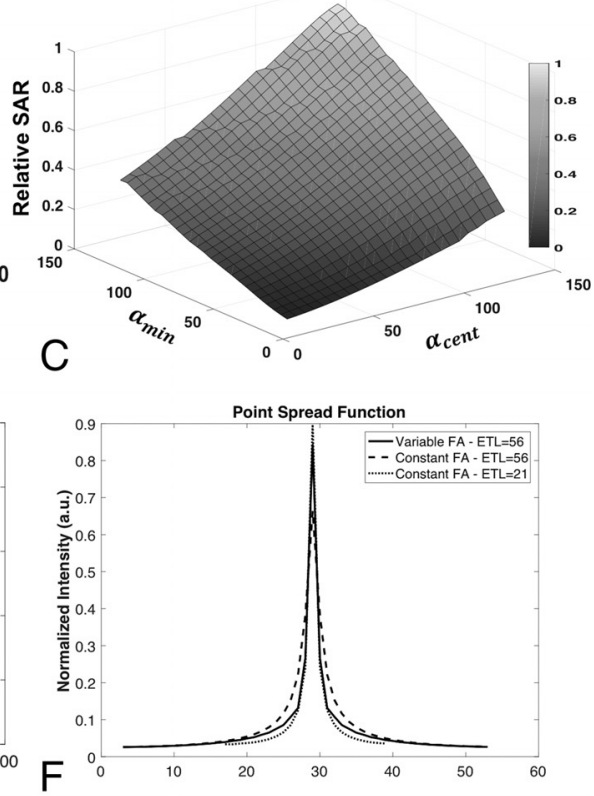

FIG 1. Surface plots of the peak and full width at half maximum (FWHM) of the simulated point spread function (PSF) as a function of $\alpha_{\text {min }}$ and $\alpha_{\text {cent }}$ are shown in $A$ and $B$, respectively. C, The relative SAR as a function of the 2 control angles. Note that the computed PSF is maximized at higher values of $\alpha_{\text {cent }}$, however, at the cost of increased SAR. D. The refocusing flip angle modulation scheme for a conventional fast spin-echo and the variable flip angle sequence along with the T2 signal evolution (E). Note that the VFA scheme stabilizes the signal evolution over the echo-train. The point spread functions for the constant and the variable flip angle echo-trains are compared in $F$. There is a considerable improvement in the PSF with the use of variable refocusing flip angles at longer echo-train lengths, resulting in better spatial resolution and less blurring. FA indicates flip angle; deg, degree; a.u., arbitrary units.

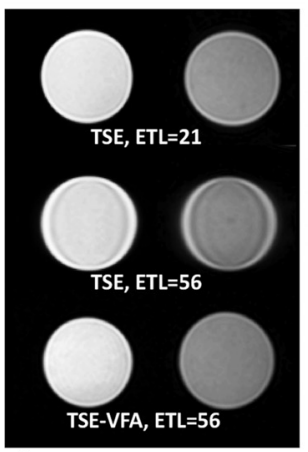

A
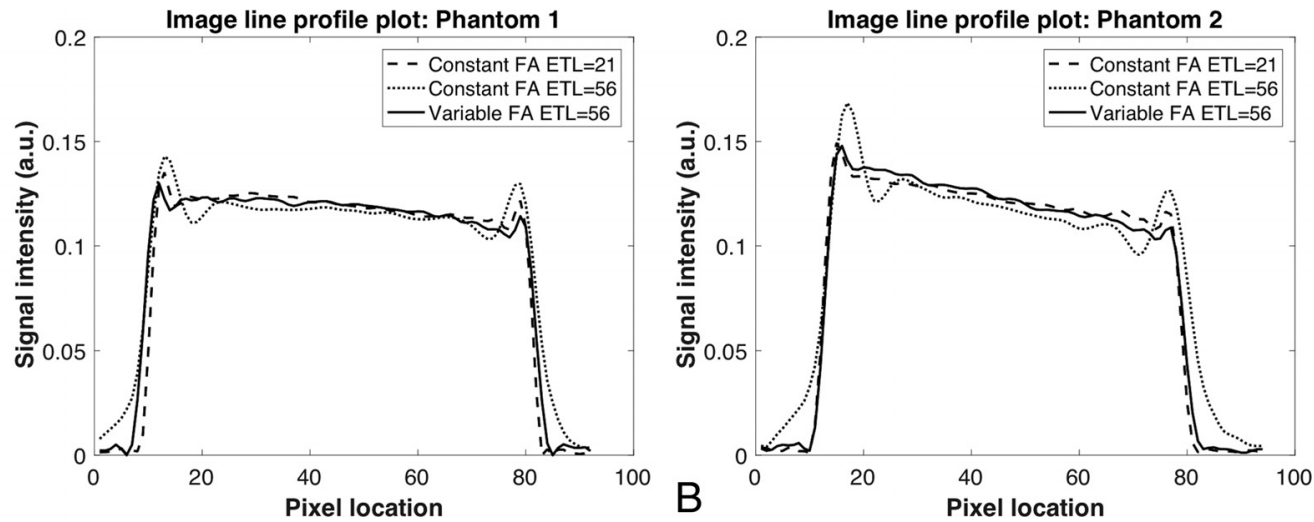

FIG 2. Phantom experiments comparing the resolution performance of TSE-VFA. Data were acquired on agarose gel phantoms $(A)$ using the conventional TSE at ETL $=21, \mathrm{ETL}=56$, and TSE-VFA at ETL $=56 . B$, Line plots across the 2 phantoms for the 3 sequences. Note the reduction in ringing when using TSE-VFA at the longer echo-train length of 56. FA indicates flip angle; a.u., arbitrary units.

TSE, TSE-VFA, and HASTE-VFA are shown in Table 1. As part of a prospective study, data were acquired from 51 patients ( 35 lumbar spine, 16 whole-spine) after written informed consent and in compliance with the institutional review board.

\section{Image-Quality Assessment}

Performance of the proposed VFA sequences was assessed independently by 2 neuroradiologists ( 2 and 12 years of experience) in a blinded fashion for both the volunteer and clinical data. To avoid recall bias, images from the 2 sequences were scored at least 2 weeks apart with random assignment of one or the other sequence for each patient in each reading session. Images were graded on a scale of $1-5$ (1, nondiagnostic images; 2 , severely limited; 3, limited; four, 1 or 2 suboptimal attributes but still diagnostic; and 5, optimal image quality for diagnosis). Images were assessed on the basis of the following criteria: edge sharpness, motion, artifacts, and noise. Clinical utility of the sequences was assessed by quantifying the ability of the reader to interpret facet joints, endplates, nerve roots, spinal cord, and discs, also on a scale of 1-5. All levels of the spine were evaluated in terms of the imagequality metrics and the degree to which each structure could be interpreted. The lowest score was then applied for each of the anatomic structures.

\section{Quantitative Analysis}

An ROI analysis was performed on the volunteer and clinical lumbar spine data to quantitatively measure the SNR and relative contrast of the 2 sequences. ROIs were placed in the L3 vertebral 
Table 2: Quantitative analysis of TSE and TSE-VFA using phantom data

\begin{tabular}{lccccccc}
\multicolumn{1}{c}{ Sequence } & $\begin{array}{c}\text { SNR } \\
\text { Phantom 1 }\end{array}$ & $\begin{array}{c}\text { SNR } \\
\text { Phantom 2 }\end{array}$ & $\begin{array}{c}\text { SNR Efficiency } \\
\text { Phantom 1 }\end{array}$ & $\begin{array}{c}\text { SNR Efficiency } \\
\text { Phantom 2 }\end{array}$ & $\begin{array}{c}\text { Relative } \\
\text { Contrast }\end{array}$ & $\begin{array}{c}\text { Scan } \\
\text { SAR }\end{array}$ & Time (min) \\
\hline Conventional TSE ETL $=21$ & 469.76 & 248.56 & 261.38 & 138.30 & 0.47 & 0.24 & $3 \mathrm{~min} 13 \mathrm{sec}$ \\
Conventional TSE ETL $=56$ & 412.99 & 195.29 & 201.52 & 95.29 & 0.46 & 0.38 & $4 \mathrm{~min} 12 \mathrm{sec}$ \\
TSE-VFA ETL $=56$ & 423.54 & 216.12 & 338.02 & 172.48 & 0.46 & 0.17 & $1 \mathrm{~min} 34 \mathrm{sec}$ \\
\hline
\end{tabular}

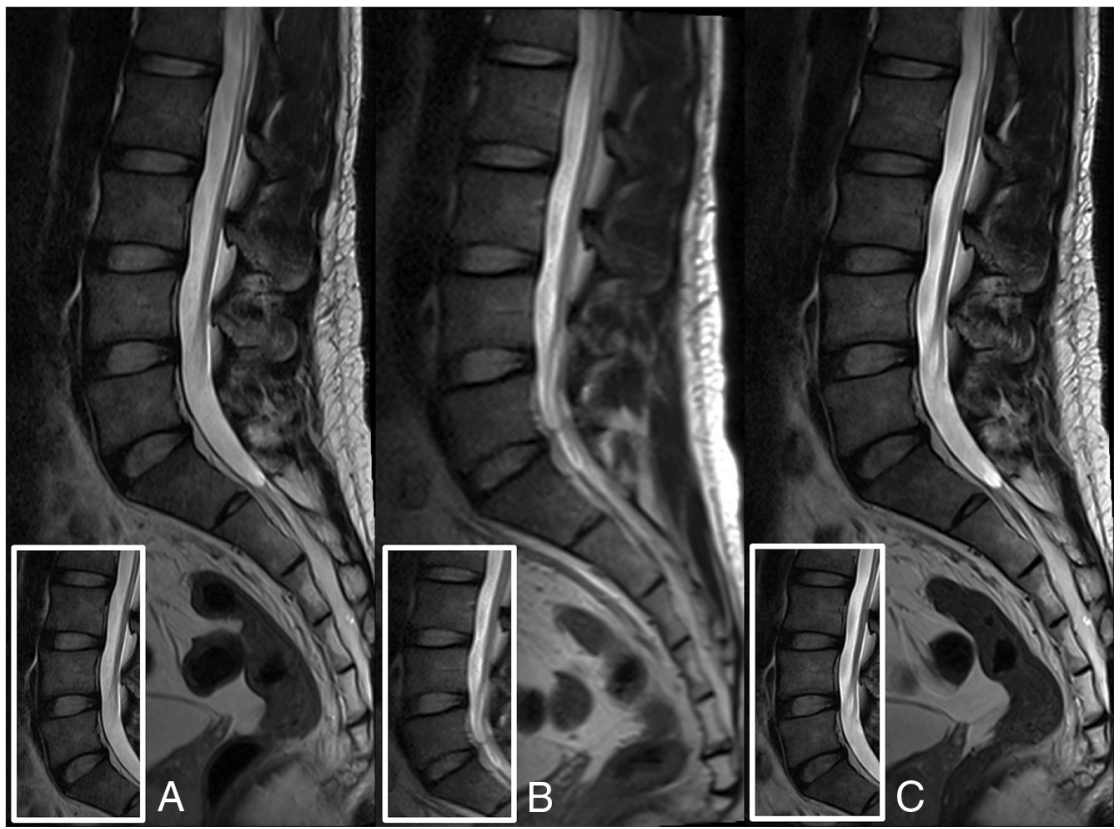

FIG 3. Images of the lumbar spine demonstrating better PSF behavior (reduced blurring) with variable flip angle TSE at ETL $=56(A)$ compared with conventional TSE at the same ETL (B). C, Image acquired using the TSE sequence at ETL $=21$. The TSE-VFA had a lower SAR value of 1.22 compared with the TSE at ETL $=21$ (SAR = 1.665). Note that at the effective TE $=105 \mathrm{~ms}$, the contrast between TSE-VFA and the T2-TSE sequence is comparable.

Table 3: Image-quality assessment scores for lumbar spine data of 5 volunteers

\begin{tabular}{lccc}
\hline \multicolumn{1}{c}{$\begin{array}{c}\text { Scoring } \\
\text { Criteria }\end{array}$} & $\begin{array}{c}\text { Mean } \\
\text { Score TSE }\end{array}$ & $\begin{array}{c}\text { Mean Score } \\
\text { TSE-VFA }\end{array}$ & $\begin{array}{c}\text { Weighted } \\
\text { Gwet AC1 }\end{array}$ \\
\hline Motion & $4.9 \pm 0.32$ & $5 \pm 0$ & 0.97 \\
Artifacts & $5 \pm 0$ & $5 \pm 0$ & 1 \\
Edge sharpness & $5 \pm 0$ & $5 \pm 0$ & 1 \\
SNR & $5 \pm 0$ & $4.4 \pm 0.52$ & 0.93 \\
Facet joints & $5 \pm 0$ & $4.8 \pm 0.42$ & 1 \\
Endplates & $5 \pm 0$ & $5 \pm 0$ & 1 \\
Nerve roots & $5 \pm 0$ & $4.8 \pm 0.42$ & 1 \\
Spinal cord & $5 \pm 0$ & $5 \pm 0$ & 1 \\
Discs & $5 \pm 0$ & $5 \pm 0$ & 1 \\
\hline
\end{tabular}

body and the L3-L4 disc to measure the mean signal intensity. The noise SD was measured from the background air region. The vertebral body SNR was calculated as SNR $=\frac{\text { Signal }_{\text {mean }}}{\text { Noise }_{\text {stdev }}}$, and the SNR efficiency was defined as SNR per unit scan time computed as $S N R_{\text {eff }}=\frac{S N R}{\sqrt{\text { Scan Time }}}$. The relative contrast between the disc and the vertebral body was calculated to quantify image contrast, defined as Contrast ${ }_{\text {vertebrae-disc }}=\frac{\text { Signal }_{\text {vertebrae }}-\text { Signal }_{\text {disc }}}{\text { Signal }_{\text {vertebrae }}}$ A 2-tailed $t$ test was performed to compare the mean SNR, relative contrast, and SAR from the 2 sequences for the 35 clinical scans.

\section{Statistical Analysis}

Noninferiority Analysis. Statistical analysis was performed using $\mathrm{R}$ statistical and computing software, Version 3.4.1 (http://www.r-project.org/). ${ }^{18}$ A noninferiority analysis between TSE-VFA and conventional TSE was performed using a 1-sided Wilcoxon signed rank test ${ }^{19}$ for the healthy volunteers and clinical patients. In this analysis, we sought to show the noninferiority of the proposed TSE-VFA compared with the conventional sequence-that is, the proposed technique does not significantly differ from the conventional method by testing the following hypothesis: $H_{0}: M_{\mathrm{VFA}}-$ $M_{\mathrm{TSE}} \leq-\Delta ; H_{\mathrm{a}}: M_{\mathrm{VFA}}-M_{\mathrm{TSE}}>-\Delta$, where $M_{\mathrm{TSE}}$ and $M_{\mathrm{VFA}}$ are the median scores of the 2 sequences respectively, $\mathrm{H}_{0}$ is the null hypothesis and $\mathrm{H}_{\mathrm{a}}$ is the alternate hypothesis. The clinically acceptable range of differences is defined using the noninferiority margin $\Delta$. In this context, the null hypothesis states that the median difference in image quality scores between TSE and TSE-VFA is greater than a predetermined noninferiority margin $\Delta$. The null hypothesis implies differences in the performance between the 2 sequences, and by rejecting the null at the significance level $(\alpha)$, we show noninferiority in the performance of TSE-VFA compared with TSE. The analysis was performed with a noninferiority margin of $\Delta=0.5$ and significance level $\alpha=.025$. The noninferiority margin $\Delta=0.5$ was chosen on the basis of preliminary data using recommendations from $\mathrm{Ahn}$ et $\mathrm{al}^{19}$ for noninferiority studies in radiology. To reduce type I error from multiple comparisons, we adjusted $P$ values from the hypothesis test with the Bonferroni correction factor of 9. A $P$ value $<\alpha$ results in the rejection of the null hypothesis and implies noninferiority of the proposed sequence compared with conventional TSE.

\section{Agreement Analysis}

To compare the clinical utility of the 2 sequences, we performed an agreement analysis ${ }^{20,21}$ using TSE as the non-reference standard for the 5 clinical criteria considered (ie, facet joints, endplates, nerve roots, spinal cord, and discs). A McNemar test of proportions was performed for each of the clinical criteria on the basis of the assumption that a score of $\geq 4$ indicates diagnostic image quality. In accordance with FDA statistical guidelines, ${ }^{21}$ an overall percentage agreement and the positive percentage agreement were also computed to characterize the diagnostic agreement of the proposed TSE-VFA sequence with TSE. 
Table 4: Quantitative analysis of lumbar spine data from volunteers and clinical subjects

\begin{tabular}{llccrr}
\hline & Sequence & $\begin{array}{c}\text { Vertebral } \\
\text { Body SNR }\end{array}$ & $\begin{array}{c}\text { Vertebral Body } \\
\text { SNR Efficiency }\end{array}$ & \multicolumn{2}{c}{$\begin{array}{c}\text { Vertebrae-Disc } \\
\text { Relative Contrast }\end{array}$} \\
\hline Healthy volunteers & TSE & $36.08 \pm 7.51$ & $19.48 \pm 4.05$ & $0.47 \pm 0.33$ & $1.69 \pm 0.14$ \\
& TSE-VFA & $27.81 \pm 4.33$ & $22.94 \pm 3.57$ & $0.45 \pm 0.32$ & $1.31 \pm 0.21$ \\
Clinical patients & TSE & $55.45 \pm 18.75$ & $29.93 \pm 10.12$ & $0.13 \pm 0.69$ & $1.84 \pm 0.61$ \\
& TSE-VFA & $45.18 \pm 14.96$ & $37.27 \pm 12.34$ & $0.12 \pm 0.7$ & $1.37 \pm 0.34$ \\
\hline
\end{tabular}

The Cohen $\kappa$ statistic was initially used to assess interobserver reliability. However, it was observed that the statistic yielded low values for grading criteria that have a high percentage of agreement and are skewed in the score distribution. To overcome this so-called $\kappa$ paradox, ${ }^{22}$ we instead used a weighted Gwet AC1 statistic to measure interobserver variability because it has been shown ${ }^{23}$ to be more robust to skewed distributions. For HASTE-VFA, images were graded by the 2 observers and the Gwet $\mathrm{AC} 1$ was computed.

\section{RESULTS}

Figure 1 shows surface plots of the peak and full width at half maximum of the point spread function and SAR computed from the simulated TSE signal evolution as a function of $\alpha_{\min }$ and $\alpha_{\text {cent. }}$. For a fixed echo-train length and effective TE, a lower $\alpha_{\min }$ and higher $\alpha_{\text {cent }}$ results in better peak signal and resolution, with a very small increase in SAR. Figure 1 also illustrates a VFA scheme and compares the simulated T2 signal decay curves for the constant and proposed VFA echo-trains. From the point spread function plots in Fig $1 F$, the improvement in spatial resolution with VFA echo-trains can be clearly observed at longer ETLs, albeit with a slight reduction in signal compared with a short ETL constant flip angle sequence.

T2WI generated using the agarose gel phantoms is shown in Fig $2 A$. To visualize the spatial resolution differences between the acquisitions, a line profile through the 2 phantoms is shown in Fig $2 B$. The conventional TSE sequence with a constant flip angle exhibits ringing artifacts at long echo-train lengths as observed in the acquired images and the line plots. Table 2 shows the SNR and relative contrast values for the 3 different sequences along with the SAR value computed by the scanner. The proposed TSE-VFA has a $1.5 \times$ reduction in SAR compared with the short ETL conventional TSE sequence and $2.3 \times$ reduction compared with the long ETL variant. The proposed sequence can generate images with comparable contrast in a $2 \times$ shorter scan time. While prolongation of the echo-train and insufficient magnetization recovery lead to a slight loss in SNR, the reduction in scan time results in slightly improved SNR efficiency.

To verify the improvement in point spread function behavior in vivo, we acquired lumbar spine data from 5 healthy volunteers using both TSE and TSE-VFA with an ETL of 56. The conventional TSE sequence with a shorter ETL of 21 was also acquired as a reference. As seen in Fig 3, TSE-VFA has considerably reduced blurring compared with TSE with ETL $=56$ and comparable image quality with TSE with ETL $=21$. The TSE-VFA also had a $1.6 \times$ lower SAR, resulting in scan time reduction from 3.9 to 1.7 minutes. With a long ETL, conventional TSE has poor image quality and higher SAR, resulting in longer scan times (3 min 12 sec) compared with TSE-VFA.

Qualitative assessment of images from 5 healthy volunteers by neuroradiologists (Table 3) showed that TSE-VFA had comparable mean scores for all the different criteria except SNR. Table 4

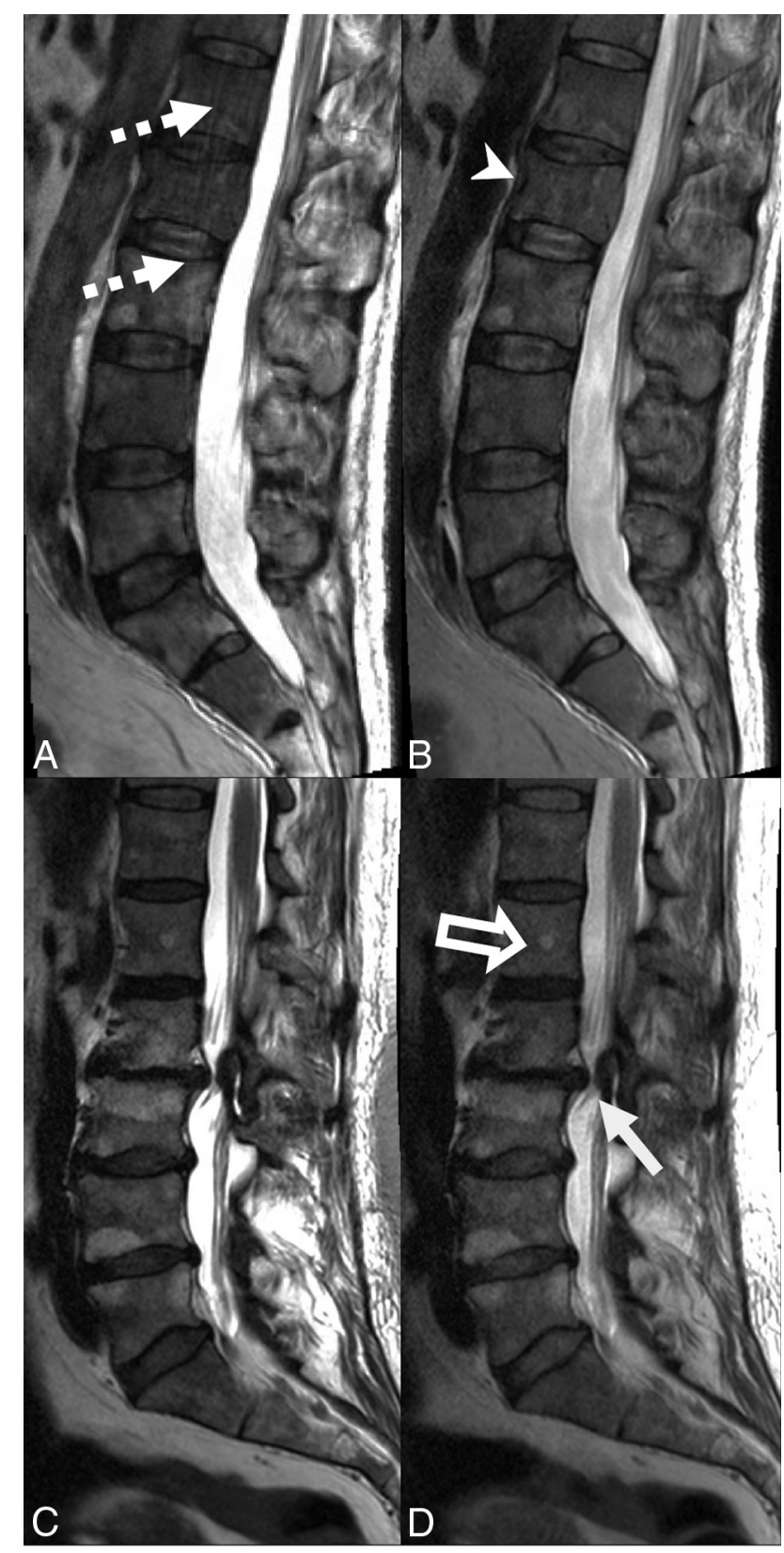

FIG 4. Sagittal T2WI TSE $(A$ and $C$ ) and TSE-VFA ( $B$ and $D$ ) images of the lumbar spine for 2 subjects. $A$ and $B$, The presence of multifocal osseous metastases with a pathologic fracture of L2 (arrowhead). The conventional TSE image ( 3 min $24 \mathrm{sec}$ scan time) shows aliasing artifacts (dotted arrows) due to motion, which are absent in the TSE-VFA image (1 min $30 \mathrm{sec}$ scan time). $C$ and $D$, Images are from a patient with multilevel degenerative disc disease and a right subarticular disc protrusion abutting the right $\mathrm{L} 3$ nerve root at $\mathrm{L} 2-\mathrm{L} 3$ (white arrow). Note that the small hemangioma within the Ll vertebral body (open arrow) is well-resolved by the TSE-VFA (D). The TSE-VFA image for this subject received a score of 4 for the SNR and the CSF signal compared with a score of 5 for the TSE image. 
Table 5: Image-quality assessment scores from 35 clinical lumbar spine cases

\begin{tabular}{lcccc}
\hline Scoring Criteria & $\begin{array}{c}\text { Mean } \\
\text { Score TSE }\end{array}$ & $\begin{array}{c}\text { Mean Score } \\
\text { TSE-VFA }\end{array}$ & $\begin{array}{c}\text { P Value of } \\
\text { Wilcoxon Test }^{\mathrm{a}}\end{array}$ & $\begin{array}{c}\text { Weighted } \\
\text { Gwet AC1 }^{2}\end{array}$ \\
\hline Motion & $4.71 \pm 0.59$ & $4.83 \pm 0.42$ & $<.001$ & 0.88 \\
Artifacts & $4.94 \pm 0.23$ & $4.76 \pm 0.46$ & $<.001$ & 0.93 \\
Edge sharpness & $4.91 \pm 0.33$ & $4.79 \pm 0.41$ & $<.001$ & $.371^{\mathrm{b}}$ \\
SNR & $4.84 \pm 0.40$ & $4.36 \pm 0.64^{\mathrm{b}}$ & $<.001$ & 0.93 \\
Facet joints & $4.83 \pm 0.40$ & $4.67 \pm 0.50$ & $<.001$ & 0.84 \\
Endplates & $4.94 \pm 0.23$ & $4.83 \pm 0.38$ & $<.001$ & 0.96 \\
Nerve roots & $4.76 \pm 0.49$ & $4.51 \pm 0.58$ & $<.01$ & 0.78 \\
Spinal cord & $4.73 \pm 0.51$ & $4.47 \pm 0.61$ & $<.001$ & 0.86 \\
Discs & $4.87 \pm 0.44$ & $4.80 \pm 0.44$ & 0.91 \\
\hline
\end{tabular}

${ }^{a}$ The null hypothesis states that the median difference in the image-quality scores between TSE and TSE-VFA is greater than the noninferiority margin $\Delta$, and rejecting the null hypothesis shows noninferiority in performance.

${ }^{b}$ Refers to lack of noninferiority between the TSE-VFA and TSE at a significance level of $P<.025$.

Table 6: Estimate of agreement in diagnostic quality between the conventional TSE and the proposed sequence

\begin{tabular}{lcc}
\hline \multirow{2}{*}{$\begin{array}{c}\text { Clinical } \\
\text { Diagnostic } \\
\text { Criteria }\end{array}$} & $\begin{array}{c}c \\
\text { Overall } \\
\text { Agreement (\%) }\end{array}$ & $\begin{array}{c}\text { Positive } \\
\text { Agreement (\%) }\end{array}$ \\
\hline Facet joints & 100 & 100 \\
Endplates & 100 & 100 \\
Nerve roots & 98.57 & 98.53 \\
Spinal cord & 97.14 & 97.06 \\
Discs & 100 & 100 \\
\hline
\end{tabular}

shows mean values from the ROI analysis on the volunteer images. The proposed sequence generates T2WI with contrast comparable with that of conventional TSE at a lower SAR. However, TSE-VFA had slightly lower measured SNR, consistent with the qualitative scores and the phantom experiments.

Figure 4 shows representative examples of lumbar spine T2WI acquired from 2 subjects using TSE and TSE-VFA sequences. Figure $4 A$ illustrates the presence of motion artifacts on a patient diagnosed with multifocal osseous metastases during the 3 min 24 sec TSE scan. On the other hand, TSE-VFA (1 min $30 \mathrm{sec}$ scan time) generated images with comparable image quality and reduced motion artifacts. Figure $4 C,-D$ shows images from a subject with multilevel degenerative disc disease. The shorter TSEVFA sequence $(D)$ has resolution comparable with that of the TSE but received a SNR score of 4 , while TSE received a score of 5. Note a slight reduction in CSF signal due to insufficient recovery of the magnetization in TSE-VFA.

Table 5 summarizes the mean scores from the 2 observers for TSE and TSE-VFA for lumbar examinations. The median difference in scores between the 2 sequences for lumbar scans was significantly $(P<.025)$ less than the noninferiority margin $(\Delta=0.5)$ for all criteria except SNR, implying noninferiority of TSE-VFA compared with conventional TSE. Note that our null hypothesis states that median difference in the image-quality scores between TSE and TSE-VFA is greater than $\Delta$. The interobserver reliability AC1 scores between readers listed in Table 5 indicate a strong agreement between the 2 readers.

The clinical utility of the TSE-VFA spine protocols is further affirmed by the high positive percentage agreement $(>80 \%)$ and overall agreement values shown in Table 6 for the lumbar spine. In addition, the McNemar test of proportions showed no significant difference $(P>.05)$ between the conventional and the proposed sequences for the clinical criteria.

The SNR and relative contrast for the lumbar spine were mea- sured for 35 clinical subjects as shown in Table 4. There was a difference in the mean SNR and relative contrast between clinical subjects and healthy volunteers for both TSE and TSE-VFA, presumably due to the hyperintense vertebral body signal caused by edema. There was no significant difference $(P=.32)$ in the vertebrae-disc relative contrast between TSE-VFA and TSE. The proposed sequence had a significantly lower $(P<.001)$ SAR compared with TSE. Note that the mean SAR for TSE was higher in clinical subjects than in healthy volunteers due to higher variability in patient weight. Furthermore, the TSE sequence had a significantly $(P=.006)$ higher SNR than TSE-VFA.

Figure 5 illustrates the utility of the fast HASTE-VFA sequence in a whole-spine protocol acquired in $<2$ minutes. While HASTE-VFA has a slightly lower resolution $(1.25 \times 1.0 \mathrm{~mm}$ compared with TSE with $1.18 \times 0.82 \mathrm{~mm}$ ), it is about 4 times faster than the TSE, making it viable in a clinical setting and potentially more robust to patient motion. HASTE-VFA (Fig 5B) clearly depicts pathology while exhibiting reduced motion artifacts in the cervical spine section compared with the 9-minute STIR-TSE scan (Fig 5A).

Mean scores for whole-spine scans along with the Gwet AC1 for interobserver agreement are shown in Table 7. There is excellent interobserver agreement in the scores except for SNR, which shows a moderate concordance. The image scores follow a trend similar to that of the lumbar TSE-VFA scores.

\section{DISCUSSION}

We have demonstrated the use of variable refocusing flip angle TSE as a fast adjunct for routine spine imaging. Flip angle modulation reduced the blurring and SAR typically associated with long-ETL TSE sequences. The TSE-VFA sequence was roughly $2.3 \times$ faster than the conventional TSE sequence $(1 \mathrm{~min} 28 \mathrm{sec}$ versus $3 \mathrm{~min} 25 \mathrm{sec}$ ). The refocusing flip angles of the TSE-VFA sequence were optimized to maximize the spatial resolution and minimize SAR. Image scores were $\geq 4$ for all the image quality and clinical criteria used and for all the clinical criteria considered; TSE-VFA was noninferior to TSE, attesting to the equivalence of the 2 sequences. While we have shown the clinical viability of TSE-VFA for sagittal T2WI, this technique can also be used as a fast alternative to the conventional TSE to generate T1WI and STIR contrasts in axial and sagittal planes. A spine protocol encompassing TSE-VFA for T1, T2, and STIR contrasts would reduce overall scan times.

We have also demonstrated the use of variable refocusing flip 


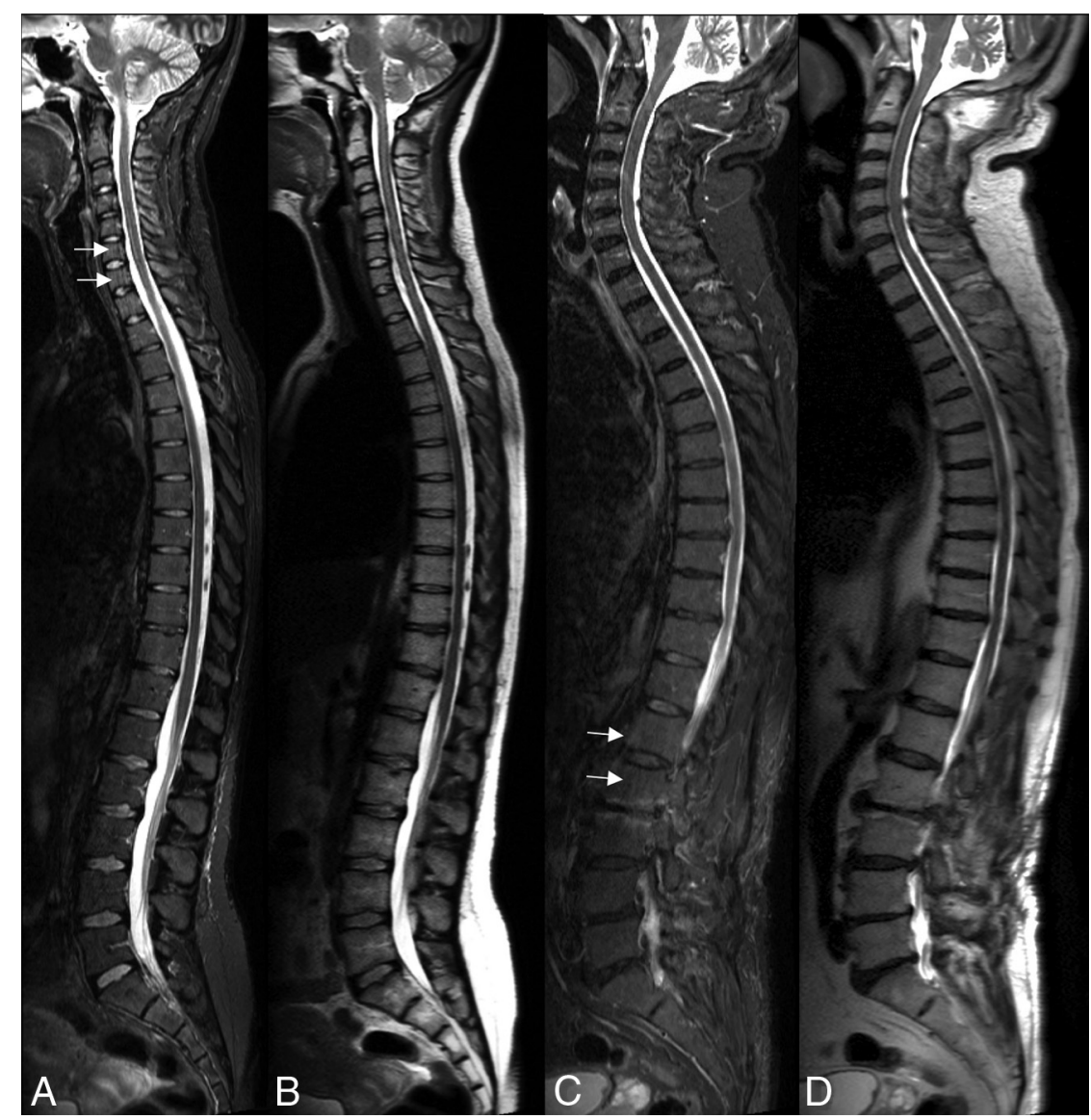

FIG 5. Sagittal STIR TSE $(A$ and $C$ ) and sagittal HASTE-VFA ( $B$ and $D$ ) images of the whole spine for 2 subjects. $A$ and $B$, Disc protrusions in the lower thoracic spine with several Schmorl nodes in the lumbar spine. $C$ and $D$, Images from a subject with degenerative disc changes in the lumbar and the lower thoracic spine. Note the increased motion-related artifacts (arrows) with the 9 min 30 sec STIR TSE sequence ( $A$ and $C$ ) when compared to the single-shot sequence ( $B$ and $D$ ) with a 1 min 54 sec scan time.

Table 7: Image-quality assessment scores for the whole-spine cases

\begin{tabular}{lcc}
\hline \multicolumn{1}{c}{$\begin{array}{c}\text { Scoring } \\
\text { Criteria }\end{array}$} & $\begin{array}{c}\text { Mean Score } \\
\text { HASTE-VFA }\end{array}$ & $\begin{array}{c}\text { Gwet ACl } \\
\text { Interobserver } \\
\text { Reliability }\end{array}$ \\
\hline Motion & $4.66 \pm 0.48$ & 0.74 \\
Artifacts & $4.56 \pm 0.50$ & 0.65 \\
Edge sharpness & $4.78 \pm 0.42$ & 0.94 \\
SNR & $4.34 \pm 0.75$ & 0.39 \\
Facet joints & $4.69 \pm 0.47$ & 0.77 \\
Endplates & $4.91 \pm 0.51$ & 0.98 \\
Nerve roots & $4.47 \pm 0.57$ & 0.66 \\
Spinal cord & $4.72 \pm 0.46$ & 0.79 \\
Discs & $4.75 \pm 0.44$ & 0.91 \\
\hline
\end{tabular}

angle single-shot sequences as a valuable addition for whole-spine imaging protocols $(4 \times$ shorter scan time), whereas TSE sequences are prohibitively long. The HASTE-VFA sequence was added to the whole-spine protocol as a fast T2WI scan ( 1 min 54 $\mathrm{sec}$ ), and its diagnostic value was confirmed by the excellent image-quality scores (clinical criteria had an average score of 4.71, and image quality metrics showed artifact behavior and SNR comparable with those in the lumbar spine). The HASTE-VFA protocol had a slightly lower resolution than the TSE-VFA $(1.25 \times 1 \mathrm{~mm}$ versus $0.81 \times 0.73 \mathrm{~mm})$ to minimize image blurring and maintain SNR, and this limits its use for high-resolution lumbar spine imaging. We are exploring the use of optimal radiofrequency pulses to further reduce echospacing and enable higher resolution single-shot $\mathrm{T} 2$ imaging.

While the mean motion score of TSE-VFA was noninferior to TSE for the clinical subjects (Table 5), presumably due to the relative lack of patient motion in lumbar spine imaging, we observed a trend toward significance when we eliminated 9 subjects with motion scores of 5 on both sequences (4.43 for TSE-VFA versus 4.04 for TSE, $P<.1$ ). This suggests that TSE-VFA could be a good alternative to conventional TSE in patients who cannot tolerate long scans or for cervical spine imaging, in which motion artifacts are common from swallowing.

One possible limitation of the proposed VFA technique is the slight reduction in CSF signal due to insufficient magnetization recovery. This needs to be studied further in a larger clinical study, even though our study showed no detrimental effects from this phenomenon and subtle changes in CSF signal in the spine were adequately visualized in the VFA sequences due to the inherent contrast between CSF and other structures such as the spinal cord and intervertebral discs.

In TSE-VFA sequences, spatial resolution is limited by the available SNR, resulting in slightly lower SNRs for the same resolution as conventional TSE. This feature is indicated both by the slightly lower qualitative SNR scores compared with the TSE sequence (4.36 versus 4.84 ) and quantitative SNR measurements (45.18 versus 55.45). However, SNR efficiency is slightly higher for TSE-VFA (37.27 versus 29.93) due to the reduced scan time. Preliminary studies on volunteers indicate that the use of anterior coil arrays, atypical in spine imaging, in addition to the posterior coil array would improve SNR and enable comparable spatial resolution between TSE-VFA and TSE. We are currently exploring the feasibility of this clinically.

In this study, the TSE-VFA protocol parameters obtained from numeric simulations have been validated using phantoms and healthy volunteers and then tested on clinical patients. In the patient data, we did see our VFA protocol perform comparably with the conventional TSE, indicating that we are not far off from the volunteer and phantom optimal values. However, an analysis on a larger cohort of clinical patients is necessary to study the optimality of the proposed protocol and its clinical diagnostic utility.

VFA sequences have also been shown ${ }^{17}$ to be sensitive to motion, a feature determined by the choice of the flip angle parameters. While low values of $\alpha_{\text {min }}$ can enable longer echo-trains, they can cause signal dephasing in the presence of substantial motion. 
VFA-based sequences are also sensitive to $B_{1}$ field homogeneities ${ }^{24}$ because $B_{1}$ variations cause changes in the refocusing flip angle that could lead to SNR loss in very large patients. We used $\alpha_{\text {min }}$ values of $45^{\circ}-50^{\circ}$, which were a good compromise between blurring and motion sensitivity.

\section{CONCLUSIONS}

We have developed fast T2WI spine protocols using variable refocusing flip angles, including a single-shot variant. VFA-based sequences have better point spread function behavior than their constant flip angle counterparts and are less sensitive to patient motion, often observed in longer TSE scans. These features enable the use of VFA sequences as an adjunct or replacement in clinical spine protocols, especially for pediatric $3 \mathrm{~T}$ spine imaging where TSE scans are highly SAR limited.

\section{ACKNOWLEDGMENTS}

The authors thank Dr Srinivasan Vedantham for helping with the statistical analysis and interpretation of the results.

Disclosures: Manojkumar Saranathan-UNRELATED: Consultancy: ABC Medical Education.

\section{REFERENCES}

1. Perry J, Haughton V, Anderson PA, et al. The value of T2 relaxation times to characterize lumbar intervertebral disks: preliminary results. AJNR Am J Neuroradiol 2006;27:337-42 Medline

2. Stankiewicz JM, Neema M, Alsop DC, et al. Spinal cord lesions and clinical status in multiple sclerosis: a 1.5T and 3T MRI study. J Neurol Sci 2009;279:99-105 CrossRef Medline

3. Fruehwald-Pallamar J, Szomolanyi P, Fakhrai N, et al. Parallel imaging of the cervical spine at 3T: optimized trade-off between speed and image quality. AJNR Am J Neuroradiol 2012;33:1867-74 CrossRef Medline

4. O'Connell MJ, Ryan M, Powell T, et al. The value of routine MR myelography at MRI of the lumbar spine. Acta Radiol 2003;44: 665-72 Medline

5. Duerinckx AJ, Yu WD, El-Saden S, et al. MR imaging of cervical spine motion with HASTE. Magn Reson Imaging 1999;17:371-81 CrossRef Medline

6. Xie C, Kong K, Guan J, et al. SSFSE sequence functional MRI of the human cervical spinal cord with complex finger tapping. Eur $J$ Radiol 2009;70:1-6 CrossRef Medline

7. Greenspan SL, Mathews VP, Caldemeyer KS, et al. FLAIR and HASTE imaging in neurologic diseases. Magn Reson Imaging Clin $N$ Am 1998;6:53-65 Medline

8. Hennig J, Scheffler K. Hyperechoes. Magn Reson Med 2001;46:6-12 CrossRef Medline

9. Hennig J, Weigel M, Scheffler K. Multiecho sequences with variable refocusing flip angles: optimization of signal behavior using smooth transitions between pseudo steady states (TRAPS). Magn Reson Med 2003;49:527-35 CrossRef Medline

10. Busse RF, Hariharan $\mathrm{H}, \mathrm{Vu} \mathrm{A}$, et al. Fast spin echo sequences with very long echo trains: design of variable refocusing flip angle schedules and generation of clinical T2 contrast. Magn Reson Med 2006; 55:1030-37 CrossRef Medline

11. Busse RF, Brau A, Vu A, et al. Effects of refocusing flip angle modulation and view ordering in 3D fast spin echo. Magn Reson Med 2008;60:640-49 CrossRef Medline

12. Mugler JP 3rd. Optimized three-dimensional fast-spin-echo MRI. $J$ Magn Reson Imaging 2014;39:745-67 CrossRef Medline

13. Kijowski R, Davis KW, Woods MA, et al. Knee joint: comprehensive assessment with $3 \mathrm{D}$ isotropic resolution fast spin-echo MR imaging-diagnostic performance compared with that of conventional MR imaging at 3.0 T. Radiology 2009;252:486-95 CrossRef Medline

14. Li CQ, Chen W, Rosenberg JK, et al. Optimizing isotropic threedimensional fast spin-echo methods for imaging the knee. J Magn Reson Imaging 2014;39:1417-25 CrossRef Medline

15. Lee S, Jee WH, Jung JY, et al. MRI of the lumbar spine: comparison of $3 \mathrm{D}$ isotropic turbo spin-echo SPACE sequence versus conventional 2D sequences at 3.0 T. Acta Radiol 2015;56:174-81 CrossRef Medline

16. Loening AM, Saranathan M, Ruangwattanapaisarn N, et al. Increased speed and image quality in single-shot fast spin echo imaging via variable refocusing flip angles. J Magn Reson Imaging 2015;42: 1747-58 CrossRef Medline

17. Litwiller DV, Holmes JH, Saranathan M, et al. Sensitivity of modulated refocusing flip angle single-shot fast spin echo to impulsive cardiac-like motion. In: Proceedings of the Annual Meeting of the International Society for Magnetic Resonance in Medicine and the European Society for Magnetic Resonance in Medicine, Milan, Italy. May $10-16,2014$

18. R Core Team. R: A Language and Environment for Statistical Computing. Vienna: R Foundation for Statistical Computing; 2013

19. Ahn S, Park SH, Lee KH. How to demonstrate similarity by using noninferiority and equivalence statistical testing in radiology research. Radiology 2013;267:328-38 CrossRef Medline

20. Langenbucher J, Labouvie E, Morgenstern J. Measuring diagnostic agreement. J Consult Clin Psychol 1996;64:1285-89 CrossRef Medline

21. Food Drug Administration Center for Devices and Radiological Health. Statistical Guidance on Reporting Results from Studies Evaluating Diagnostic Tests, Document 1620, Maryland, 2007. https:// www.fda.gov/downloads/MedicalDevices/DeviceRegulationand Guidance/GuidanceDocument/ucm071287.pdf. Accessed July 23, 2017

22. Cicchetti DV, Feinstein AR. High agreement but low kappa, II: resolving the paradoxes. J Clin Epidemiol 1990;43:551-58 CrossRef Medline

23. Wongpakaran N, Wongpakaran T, Wedding D, et al. A comparison of Cohen's Kappa and Gwet's AC1 when calculating inter-rater reliability coefficients: a study conducted with personality disorder samples. BMC Med Res Methodol 2013;13:61 CrossRef Medline

24. Madhuranthakam AJ, Busse RF, Brittain JH, et al. Sensitivity of low flip angle SSFSE of the abdomen to cardiac motion. In: Proceedings of the Joint Annual Meeting of the International Society for Magnetic Resonance in Medicine and European Society for Magnetic Resonance in Medicine and Biology, Berlin, Germany. May 19-25, 2007 